\title{
PARAMETERS OF A RADIOCARBON INSTALLATION
}

\section{VLADIMIR Z. KHAIT}

\author{
85 Yermiyahy Street, Sderot 80100 Israel
}

\begin{abstract}
I aim to define instrumental parameters of a radiocarbon laboratory installation whereby one can estimate its precision and a maximum age up to which its measuring results are reliable. The commonly accepted factor of merit (FM) relates the precision of measurement to Poisson statistics. Unlike the FM, the proposed parameters show the extent to which a ${ }^{14} \mathrm{C}$ laboratory is affected by destabilizing factors that could cause additional measurement errors. Assuming that all destabilizing factors produce either a change in counting efficiency or additional fluctuations of the background counting rate, I have derived two parameters for consideration.
\end{abstract}

Several authors (Moljk, Drever and Curran 1957; Vinogradov et al. 1961) have suggested estimating the precision of a radiocarbon age with the help of the FM $M=S / \sqrt{B}$ (where $\mathrm{S}$ and B stand for counting rates of standard and background, respectively). Using the FM, it is then possible to compare other ${ }^{14} \mathrm{C}$ laboratories with respect to their precision and maximum age. Such a comparison is not appropriate in all cases. In some cases, investigators do not use the FM to estimate the precision of the measuring results (e.g., Ryabinin 1978; Sementsov 1970). Sementsov (1970) evaluated the precision of measurement results by estimating them based on the experimental deviation of the counting rate of the standard $\sigma_{\Sigma S}$ :

$$
\sigma_{\Sigma s^{2}}=[1 /(n-1)] \cdot \sum_{i=1}^{n}\left(S_{i}-\bar{S}\right)^{2}
$$

where

$$
\begin{aligned}
& S_{i}=\text { a counting rate of a standard in } i \text { th test } \\
& \bar{S}=\sum_{i=1}^{n} S_{i} / n=\text { an average value of counting rates in } n \text { tests. }
\end{aligned}
$$

The FM was obtained under the assumption that measurement is subject to Poisson statistics. Undoubtedly, the process of a radioactivity and the formation of background signals obey Poisson statistics. Thus, according to the properties of the Poisson distribution, one can determine a counting rate deviation called the statistical deviation ${ }^{1} \sigma_{s t}$.

$$
\sigma_{s t}^{2}=S / t
$$

where

$$
\begin{aligned}
& S=\text { counting rate of standard } \\
& t=\text { counting time }
\end{aligned}
$$

The statistical deviation is not an independent value because it is determined from the already known measured values $S$ and $t$. If, under experimental conditions for the same sample, Equations (1) and (2) are in good agreement then one may claim that the ${ }^{14} \mathrm{C}$ lab is optimal. Its instrumental errors are negligibly small and may be neglected. The agreement of the errors calculated according to Equations (1) and (2) is not always observed. Some investigators (Crevecoeur, Stricht and Capron 1959) have preferred to calculate errors according to Equation (1) in spite of the fact that it is nec-

\footnotetext{
${ }^{1}$ We use the word "deviation" hereafter implying "standard deviation" $(1 \sigma)$. We do not use the term "standard deviation" so as not to confuse it with the term "standard", which has another meaning.
} 
essary to spend additional time making repeated tests before error calculation. Many investigators find that, according to their measurements, summary (experimental) errors exceed statistical errors considerably (Crevecoeur, Stricht and Capron 1959; Currie 1972; Zavelsky 1972; Khait 1982). Since it is necessary to decrease the statistical deviation to an acceptable value, one must increase the counting time. For long counting times, it is very likely that the measurement process will be affected by external factors (e.g., temperature changes, supply voltage fluctuations), the changes of which will also result in additional fluctuations in the counting rate and as a result, instrumental errors occur. Summary fluctuations contain both instrumental and statistical fluctuations. For future work, let us assume that all factors that influence the measurement process cause either a change in the count efficiency $E$ or an additional counting rate deviation of the background. Accepting this assumption, the counting rate deviation for any ${ }^{14} \mathrm{C}$ sample will be a function of the counting rate deviation of the standard and that of the background (Khait 1982), given as

$$
\sigma_{r}{ }^{2}=\left[(R-B)^{2} /(S-B)^{2}\right] \cdot \sigma_{s}{ }^{2}+\left[1-(R-B)^{2} /(S-B)^{2}\right] \cdot \sigma_{b}{ }^{2}
$$

where

$$
\begin{aligned}
& R=\text { counting rate of any sample } \\
& S=\text { counting rate of a standard } \\
& B=\text { counting rate of a background } \\
& \sigma_{r}=\text { counting rate deviation of any sample } \\
& \sigma_{s}=\text { counting rate deviation of a standard } \\
& \sigma_{b}=\text { counting rate deviation of a background } .
\end{aligned}
$$

Comparison of the results calculated by Equation (1) and those calculated by Equation (3) shows good agreement (Khait 1982). The counting rate deviations in Equation (3) are instrumental deviations. A summary (experimental) deviation consists of the instrumental deviation $\sigma$ and the statistical deviation $\sigma_{s t}$. To the extent that these values are independent, then, in accordance with the laws of statistics and with previous studies (Crevecoeur, Stricht and Capron 1959; Currie 1972; Zavelsky 1972), the following equations express the summary deviations $\sigma_{\Sigma S}, \sigma_{\Sigma r}$ and $\sigma_{\Sigma b}$ of the three counting rates $S, R$ and $B$ incorporating instrumental and statistical deviations

$$
\begin{aligned}
& \sigma_{\Sigma s}^{2}=\sigma_{S}^{2}+S / t \\
& \sigma_{\Sigma r}^{2}=\sigma_{r}^{2}+R / t \\
& \sigma_{\Sigma b}{ }^{2}=\sigma_{b}^{2}+B / t .
\end{aligned}
$$

Equations (4.1)-(4.3) allow us to determine instrumental deviations (the first expression in the right side of the equations). However, for $\sigma_{r}$ the result given in Equation (3) is to be preferred to Equation (4.2). Equation (3) does not require repeated tests. As follows from Equations (4.1)-(4.3), the statistical deviations (the second expression in the right side of the equations) can be reduced by increasing counting time. Experience shows that with an increase of counting time it is impossible to reduce the summary (experimental) deviations $\sigma_{\Sigma s}, \sigma_{\Sigma r}$ and $\sigma_{\Sigma b}$ (Currie 1972; Zavelsky 1972). At present we cannot answer how instrumental deviation depends on counting time. To overcome this problem, we assume the same counting time for standard, sample and background is used and that they appear in the same order, i.e., standard-background-sample. By following these rules, a possible dependence of instrumental deviation on counting time can be avoided. It is then possible to calculate $\sigma_{S}$ and $\sigma_{b}$ according to Equations (4.1) and (4.3) if we determine the preliminary summary deviations $\sigma_{\Sigma s}$ and $\sigma_{\Sigma b}$ according to Equation (1). It may happen that a statistical deviation is much less than an instrumental one. In that event, we can neglect the statistical deviation. In such a case, all further reasoning will also be true for the summary (experimental) deviation as well. 
Equation (3) makes it possible to draw the following important conclusions:

1. It is possible to save counting time because there is no need to use the repeated tests to calculate the counting rate deviation for every sample.

2. It is necessary to repeat counting of the standard and background. These repeated measurements can be used in Equation (1) to calculate summary deviations.

3. It will suffice to measure the counting rate of any sample once and then calculate its deviation according to Equation (3). There is no need to use additional time for the measuring process.

The following expression allows us to calculate the deviation of a ${ }^{14} \mathrm{C}$ age.

$$
\sigma_{T}=\tau \cdot \sqrt{\sigma_{s}^{2} /(S-B)^{2}+\sigma_{r}^{2} /(R-B)+\sigma_{b}^{2} \cdot(S-R)^{2} /(S-B)^{2} \cdot(R-B)^{2}}
$$

where

$\tau=$ average time of life of ${ }^{14} \mathrm{C}$.

The investigators (Crevecoeur, Stricht and Capron 1959) used this equation for calculating the errors of a ${ }^{14} \mathrm{C}$ age. As is apparent from Equation (3), $\sigma_{r}$ is a value dependent on $\sigma_{s}$ and $\sigma_{b}$. Substituting $\sigma_{r}$ into Equation (5), according to Equation (3), we get the following transformed expression

$$
\sigma_{T}=\sqrt{2} \cdot \tau \sqrt{\sigma_{s}^{2} /(S-B)^{2}+\sigma_{b}^{2}(S-R) /(S-B) \cdot(R-B)^{2}} .
$$

Also

$$
\begin{aligned}
& (R-B)=(S-B) \cdot \exp (-T / \tau) \\
& R=(S-B) \cdot \exp (-T / \tau)+B .
\end{aligned}
$$

After substituting $(R-B)$ and $R$ of (7) into (6), the expression $\sigma_{T}$ becomes

$$
\sigma_{T}=\sqrt{2} \cdot \tau \cdot \sigma_{s} /(S-B) \cdot \sqrt{1+\exp (T / \tau) \cdot[\exp (T / \tau)-1] \cdot \sigma_{b}^{2} / \sigma_{s}^{2}} .
$$

Equation (8) is a function of the instrumental deviation of the sample's age. The behavior of Equation ( 8$)$ is determined by its coefficients

$$
\begin{gathered}
P=\sigma_{s} /(S-B) \\
Q=\sigma_{b} / \sigma_{s} .
\end{gathered}
$$

Replacing $\sigma_{s} /(S-B)$ and $\sigma_{b} / \sigma_{s}$ with their symbols, Equation (8) becomes

$$
\sigma_{T}=\sqrt{2} \tau \cdot P \sqrt{1+\exp (T / \tau) \cdot[\exp (T / \tau)-1] \cdot Q^{2}} .
$$

We may assume that the nature of the instrumental deviation of a ${ }^{14} \mathrm{C}$ age for any ${ }^{14} \mathrm{C}$ laboratory is given by Equation (10).

Two graphs in Figure 1 show the deviation as a function of age: graph 1 was carried over from a paper by Crevecoeur, Stricht and Capron (1959); graph 2 was plotted according to Equation (10) for the instrumental deviations of the liquid scintillation counter that was used in Moscow State University's Laboratory of Recent Sediments and Pleistocene Paleogeography in 1976. 


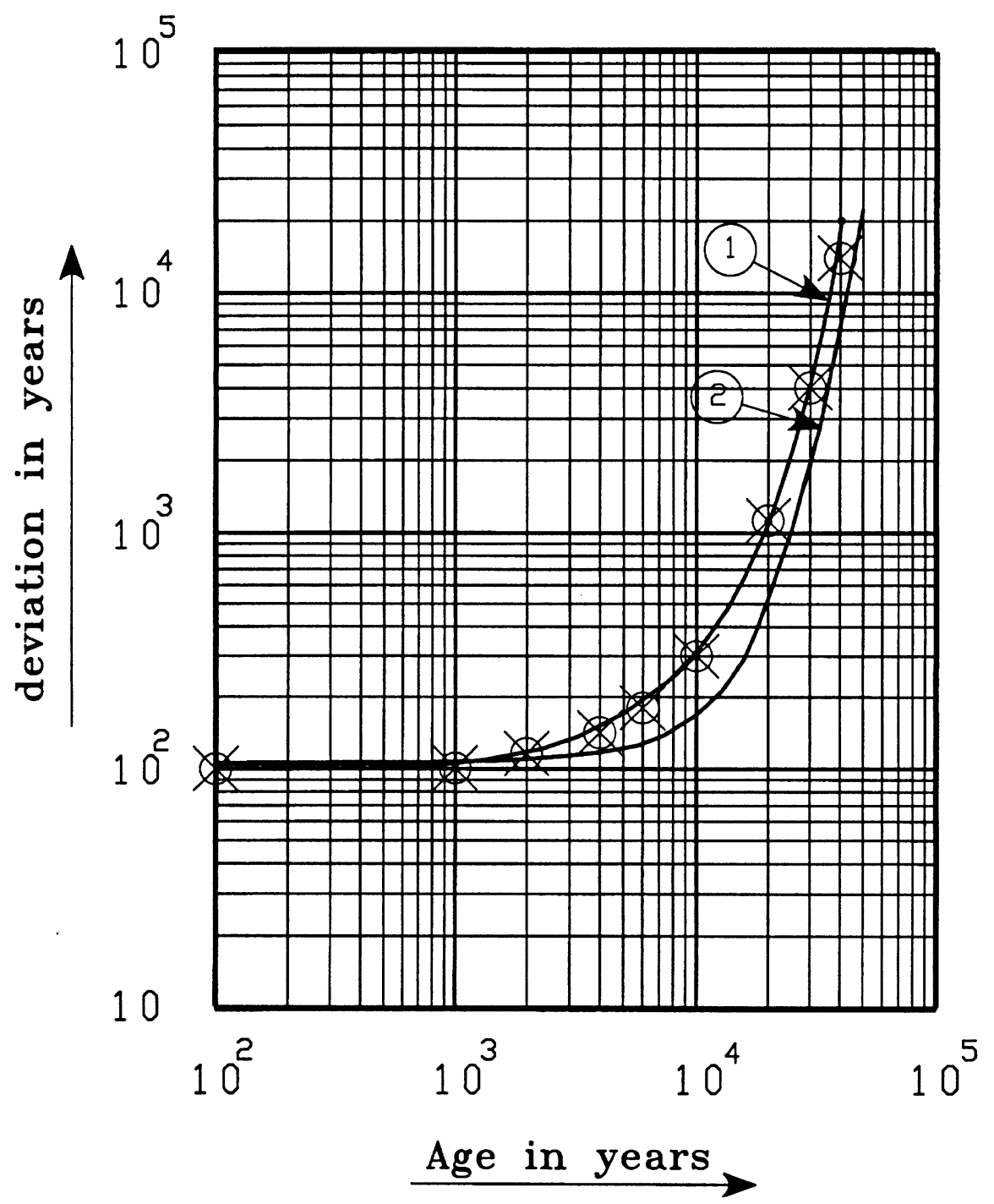

Fig. 1. Standard deviation as a function of age

From the similarity of the two graphs in Figure 1, it is very likely that our assumption mentioned above is correct. To verify this, the coefficients of the first graph were determined by solving two equations to give
1) $T=0 ; \delta_{T}=100$
2) $T=30,000 ; \delta_{T}=400$.

Despite the fact that Figure 1 (Crevecoeur, Stricht and Capron 1959) does not contain the interval of ages from $T=0$ to $T=500$, for the purpose of facilitating the calculation of its coefficients, we found it worthwhile to extend Figure 1 to intersect the error scale. In accordance with these chosen points, we have the following system of the two equations: 


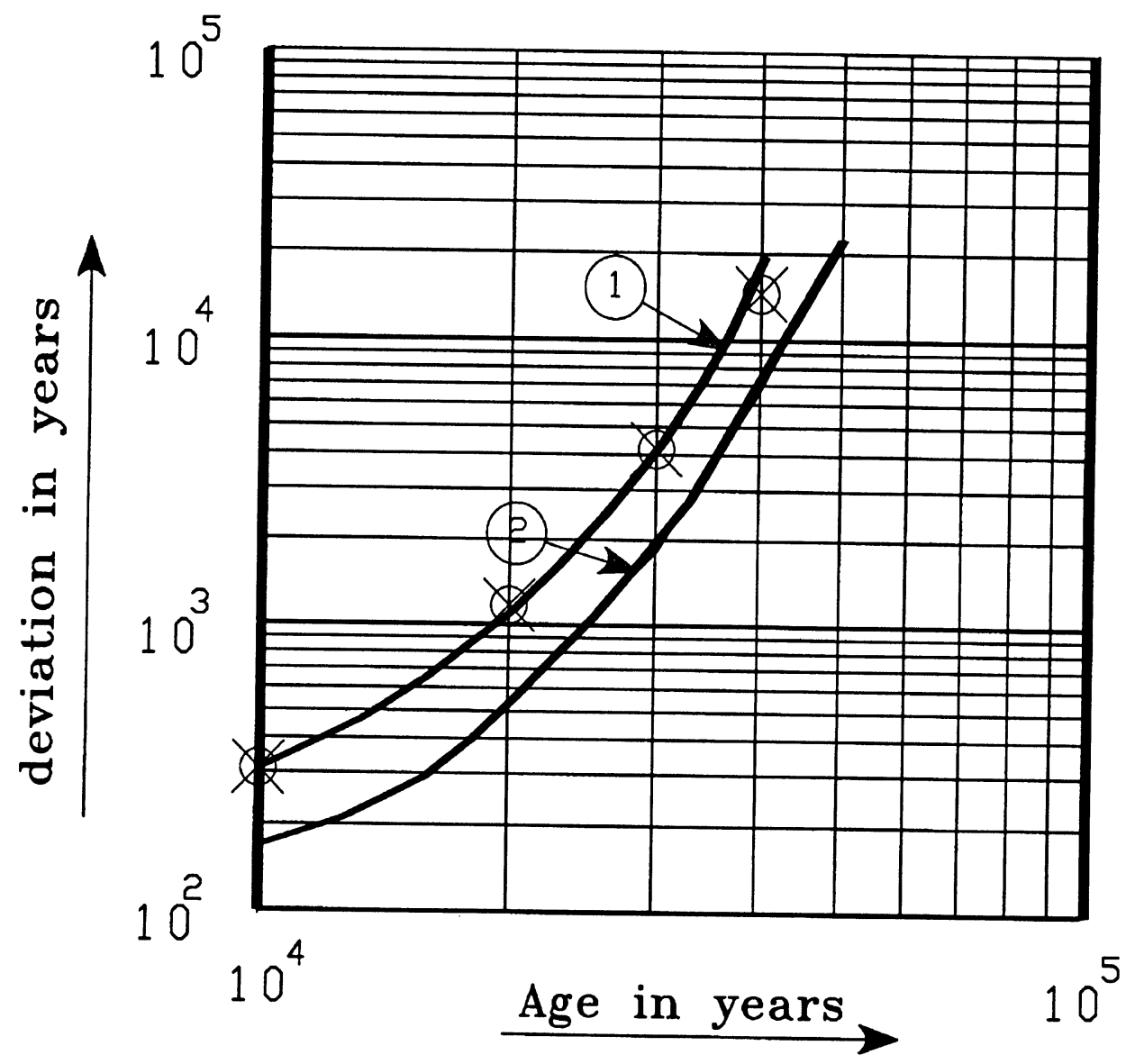

Fig. 2. Fragment of Fig. 1

$$
\begin{aligned}
100 & =\sqrt{2} \cdot \tau \cdot P \cdot \sqrt{1+\exp (0 / \tau) \cdot[\exp (0 / \tau)-1] \cdot Q^{2}} \\
4000 & =\sqrt{2} \cdot \tau \cdot P \cdot \sqrt{1+\exp (30,000 / \tau) \cdot[\exp (30,000 / \tau)-1] \cdot Q^{2}} .
\end{aligned}
$$

The solution of the system gives the results $P=8.8 \cdot 10^{-3}$ and $Q=0.95$.

Using these estimated coefficients, the expression for measuring the error on a ${ }^{14} \mathrm{C}$ age for the gas counter presented by the authors (Crevecoeur, Stricht and Capron 1959) over the whole range of ages is

$$
\sigma_{T}=100 \cdot \sqrt{1+\exp (T / \tau) \cdot[\exp (T / \tau)-1] \cdot 0.9} .
$$

From Equation (12), the locations of nine points were determined (Table 1) and marked by crosses in Figure 1. As we see, the locations of these points do not deviate considerably from graph 1 . The two functions with three deviations $\sigma_{s}, \sigma_{r}$ and $\sigma_{b}$ according to Equation (5) and also the paper (Crevecoeur, Stricht and Capron 1959) and with two deviations $\sigma_{s}$ and $\sigma_{b}$ according to Equation (12), as shown in Figure 1, coincide. This confirms the above supposition and also the fact that 
instrumental errors prevailed in the results of the measurements presented in Crevecoeur, Stricht and Capron (1959). It is possible that there are instrumental errors in most ${ }^{14} \mathrm{C}$ measurements.

Coefficients $P$ and $Q$ are the parameters of Equation (10) and are the proposed parameters for measuring counting performance of a ${ }^{14} \mathrm{C}$ lab.

Assuming that $T<<\tau$, we obtain an approximate result in accordance with (10), by neglecting the second expression under the root.

$$
\sigma_{T}=\sqrt{2} \cdot P \cdot \tau .
$$

Assuming that $T \gg>$, we get an approximate result for which the first expression under the root can be neglected

$$
\sigma_{T}=\sqrt{2} \cdot P \cdot Q \cdot \tau \cdot \exp (T / \tau) .
$$

Equation (13) does not contain the variable $T$. Consequently, instrumental deviation depends very little on the age for $T<<\tau$. This is also seen in Figure 1 (for the first graph approximately up to 1000 $\mathrm{yr}$, for the second graph up to $3500 \mathrm{yr}$ ). There is only one possibility to decrease instrumental errors over this range, namely lowering $P$.

Equation (14) for $T \gg>\tau$ shows an exponential dependence on the instrumental deviation of a ${ }^{14} \mathrm{C}$ age. As shown in Equation (14), the product of coefficients $P$ and $Q$ characterizes the precision of age determination in this interval. According to Equation (9), this product is equal to

$$
P \cdot Q=\sigma_{b} /(S-B) \text {. }
$$

We can consider this product $P \cdot Q$ as an individual parameter $A$ (this is, $A=P \cdot Q$ ) on which the precision of measurements in the range of $T \gg>\tau$ depends. In any case, as follows from Eqs. (9.1) and (15), to obtain a higher precision of measurement over the whole range of ${ }^{14} \mathrm{C}$ ages that can be measured in a lab, the counting rate of the standard must be as high as possible, or, in other words, the counting efficiency must be as high as possible. To obtain a higher precision of measurements in the interval of young ages according to Equation (9.1), it is important to reduce the counting rate deviation of the standard to a minimum.

TABLE 1. Dependence of Standard Deviation of Age on Age

\begin{tabular}{lccccccccc}
\hline \multicolumn{1}{c}{ No. } & 1 & 2 & 3 & 4 & 5 & 6 & 7 & 8 & 9 \\
\hline $\begin{array}{l}\text { Age }(T) \\
\text { in years }\end{array}$ & 0 & 1000 & 2000 & 400 & 6000 & 10,000 & 20,000 & 30,000 & 40,000 \\
$\begin{array}{l}\text { Standard } \\
\text { deviation } \\
(T) \text { in years }\end{array}$ & 100 & 107 & 115 & 140 & 176 & 296 & 1100 & 3930 & 13,750 \\
\hline
\end{tabular}

For a higher precision of measurements in the range of $T \gg>$, we must reduce the counting rate deviation of the background to a minimum according to Equation (15). The precision of measurement results depends on two instrumental parameters $P$ and $Q$ over the whole range of a ${ }^{14} \mathrm{C}$ age. These parameters show the capability of a ${ }^{14} \mathrm{C}$ laboratory to resist the effect of destabilizing factors on a measurement process. Comparison of these parameters of various ${ }^{14} \mathrm{C}$ laboratories enables one to ascertain which of them can yield a higher precision of measurement in a particular part of the age scale. 
The availability of three equations for the calculation of instrumental errors (Eqs. (10), (13) and (14)) allows us to divide the whole range of ${ }^{14} \mathrm{C}$ measurements into three intervals: young, middle and ancient ages. Equation (10) is generalized. The calculations of instrumental errors by Equation (10) are always correct for the whole range of a ${ }^{14} \mathrm{C}$ laboratory. We can consider Equation (10) as a function of the parameters $P$ and $Q$. The influence of each of these parameters on the precision of measurements varies in different places on the age scale. Therefore, it is worth dividing the whole range of ${ }^{14} \mathrm{C}$ ages into intervals. This permits us to estimate the precision of measurements with the help of approximating Equations (13) and (14) showing either a prevailing influence of one of these parameters $(P$ or $A)$ their combined influence on the precision of measurement. So, the precision of measurement in the range of "young ages" depends solely on parameter $P$, but in the range of "ancient ages" it depends solely upon parameter $A$.

In other cases, the measurement error must be calculated according to Equation (10). To determine when Equations (10), (13) and (14) apply, we solve the following equations

$$
\begin{gathered}
\sqrt{1+\exp (T / \tau) \cdot[\exp (T / \tau)-1] \cdot Q^{2}}-1=\delta \\
\frac{\sqrt{1+\exp (T / \tau) \cdot[\exp (T / \tau)-1] \cdot Q^{2}}-Q \cdot \exp (T / \tau)}{Q \cdot \exp (T / \tau)}=\delta
\end{gathered}
$$

where

$\delta=$ relative permissible difference.

The root of Equation (16) is a bound between the intervals of young and middle ages. We can find its value according to the expression

$$
T_{y m}=\tau \cdot \ln \left(0.5+\sqrt{0.25+2 \delta / Q^{2}}\right) .
$$

The root of Equation (17) is a bond between the intervals of middle and ancient ages. We can find its value according to the following expression

$$
T_{m a}=\tau \cdot \ln \left(-\frac{1}{4 \cdot \delta}+\sqrt{\frac{1}{16 \cdot \delta^{2}}+\frac{1}{2 \cdot \delta \cdot Q^{2}}}\right) .
$$

$T_{y m}$ is a bound up to which we can use Equation (13) for the calculation of instrumental errors (deviations) $\delta_{T} . T_{m a}$ is a bound over which we can use Equation (14) for the calculation of instrumental errors. Then the difference in instrumental errors between each of the approximation equations and the generalized Equation (10) will not exceed a specified relative permissible difference $\delta$. Assuming the relative permissible difference to be $10 \%(\delta=0.1), T_{y m}$ for each of the above-mentioned counters will be equal:

$$
\begin{gathered}
T_{y m}(\text { gas counter })=1397 \mathrm{yr} \\
T_{y m}(\text { scintillation counter })=4042 \mathrm{yr} .
\end{gathered}
$$

Assuming the relative permissible difference to be $10 \%(\delta=0.1)$, the bound $T_{m a}$ was determined only for the scintillation counter that was used in the ${ }^{14} \mathrm{C}$ laboratory of Moscow University in 1976. It is equal to $9520 \mathrm{yr}$. It was not possible to determine $T_{m a}$ for the gas counter used by Crevecoeur, Stricht and Capron (1959) because the relative permissible difference between the generalized 
Equation (10) and the approximation Equation (14) could not be achieved at any of the points of the range of this gas counter.

As follows from Equation (13), the precision of measurements in the range of young ages depends almost entirely on the parameter $P=\sigma_{s} /(S-B)$. The lower the value for $P$, the smaller the error of a measurement result in this interval. The bound $T_{y m}$ between the interval of young and middle ages determines the width of the interval of young ages $\left(O<T<T_{y m}\right)$. Errors of measurement in this interval are minimum and almost constant. The higher the value for $T_{y m}$, the better the lab. The locations of the bounds $T_{y m}$ in the graphs for two counters of Figure 1 are also in agreement with the fact that instrumental errors hardly ever change over the range of young ages. As follows from Equation (18), the parameter $Q=\sigma_{b} / \sigma_{s}$ influences the location of $T_{y m}$ on the age scale. Thus we can evaluate all the capabilities of a ${ }^{14} \mathrm{C}$ laboratory using its two parameters $P$ and $Q$. The growth of measurement errors becomes appreciable for $T>T_{y m}$. This fact can also be observed on the graphs of Figure 1 . The precision of measurements in the interval of middle ages $\left(T_{y m}<T<T_{m a}\right)$, as follows from the generalized Equation (10), depends on the two parameters $P$ and $Q$. As the age $T$ grows, the influence of the parameter $Q$ on the precision of dating grows too. As Equation (19) shows, the location of the bound between middle and ancient ages also depends on the parameter $Q$.

As follows from Equation (14), the instrumental error $\sigma_{\mathrm{T}}$ is an exponential function of the age $T$ for $T>T_{m a}$. The growth of measurement errors in this interval is quicker than over the central range of ages. Therefore, the higher the bound between middle and ancient ages, the better the lab. The product of the parameters $P$ and $Q$ determines the precision of dating in the interval of ancient ages according to Equation (14).

Still missing from our list of parameters is the maximum age $T_{\max }$. It is possible to deduce this important additional parameter as a function of the parameters $P$ and $Q$. However, the maximum age is the subject of separate investigation and will be dealt with in future research.

Table 2 presents the parameters and the equations for their calculation. The results of the calculations of some of these parameters are also presented in this table for two counters under discussion. We have used equations by Moljk, Drever and Curran (1957) for the calculation of the maximum age. Crevecoeur, Stricht and Capron (1959) proposed that the maximum age should be determined if there are summary (experimental) errors of measurements. Moljk, Drever and Curran (1957) suggest determining the maximum age as a function of the FM. Table 2 lists the results of the calculations of $T_{\max }$ performed in both of these ways for the scintillation counter used in 1976 at Moscow University's ${ }^{14} \mathrm{C}$ laboratory. The counting efficiency $E$ is also included in Table 2.

It is possible to express the parameters $P$ and $A$ in terms of counting efficiency

$$
\begin{aligned}
& P=\sigma_{s} / E \cdot d \cdot W \\
& A=\sigma_{b} / E \cdot d \cdot W
\end{aligned}
$$

where

$d=$ specific radioactivity

$W=$ weight of a sample .

It is possible to determine the weight of a sample to obtain the necessary values for $P$ and $A$, ensuring the required precision of measurements. 
TABLE 2. Parameters of a Radiocarbon Installation

\begin{tabular}{|c|c|c|c|c|c|}
\hline \multirow[b]{3}{*}{ No. } & \multirow[b]{3}{*}{$\begin{array}{l}\text { Parameter } \\
\text { symbol }\end{array}$} & \multirow[b]{3}{*}{ Parameter name } & \multirow[b]{3}{*}{ Calculation formula } & \multicolumn{2}{|c|}{ Type of counter } \\
\hline & & & & Gas & Scintillation \\
\hline & & & & $\begin{array}{l}\text { University of } \\
\text { Louvain }\end{array}$ & $\begin{array}{c}\text { Moscow } \\
\text { University }\end{array}$ \\
\hline 1 & $E(\%)$ & Counting efficiency & $\frac{S-B}{d \cdot w} \cdot 100$ & -- & 27 \\
\hline 2 & $M$ & FM & $S / \sqrt{B}$ & -- & 28 \\
\hline 3 & $P$ & & $\sigma_{s} /(S-B)$ & $8.8 \times 10^{-3}$ & $9.33 \times 10^{-3}$ \\
\hline 4 & $Q$ & & $\sigma_{s} / \sigma_{B}$ & 0.95 & 0.42 \\
\hline 5 & $A$ & & $P \cdot Q=\sigma_{B} /(S-B)$ & $8.36 \times 10^{-3}$ & $3.92 \times 10^{-3}$ \\
\hline 6 & $T_{y m}(\mathrm{yr})$ & $\begin{array}{l}\text { Bound between } \\
\text { intervals of young } \\
\text { and middle ages }\end{array}$ & $\tau \cdot \ln \left(0.5+\sqrt{0.25+\frac{2 \cdot \delta}{Q^{2}}}\right)$ & 1397 & 4042 \\
\hline 7 & $T_{m a}(\mathrm{yr})$ & $\begin{array}{l}\text { Bound between } \\
\text { intervals of middle } \\
\text { and ancient ages } \\
\end{array}$ & $\tau \cdot \ln \left(-\frac{1}{4 \cdot \delta}+\sqrt{\frac{1}{16 \cdot \delta^{2}}+\frac{1}{2 \cdot \delta \cdot Q^{2}}}\right)$ & -- & 9520 \\
\hline \multirow[t]{2}{*}{8} & \multirow[t]{2}{*}{$T_{\max }(\mathrm{yr})$} & \multirow[t]{2}{*}{ Maximum age } & $\tau \cdot \ln \frac{S-B}{\sqrt{2} \cdot \delta_{B}}$ & $\cdots$ & 43,360 \\
\hline & & & $\tau \cdot \ln \left(\frac{1}{6} M \sqrt{t}\right)$ & -- & 40,500 \\
\hline
\end{tabular}

\section{CONCLUSION}

We have obtained a system of instrumental parameters for a ${ }^{14} \mathrm{C}$ lab as a result of an investigation of instrumental errors of measurements. Two of these parameters, $P$ and $A$, are fundamental. They are fundamental because they will suffice to determine completely the precision of measurements over the whole age range capability of a ${ }^{14} \mathrm{C}$ laboratory. We can replace the parameter $Q$ with the parameter $A$ according to the formula $A=P Q$. Then the two parameters $P$ and $A$ will be fundamental. Additional parameters, such as the bounds of age ranges and the maximum age, enable us to evaluate quickly the performance of a ${ }^{14} \mathrm{C}$ lab. The FM is also a fundamental parameter as the precision of measurements is directly dependent upon it. This parameter is suitable for an ideal case only, that is, for measurements without fluctuations in counting efficiency and without additional fluctuations of background, and therefore I propose that $P$ and $A$ should also be considered fundamental.

The rest of the parameters are additional, being related to the fundamental ones in a certain way. The additional parameters enable us to quickly evaluate the performance of a ${ }^{14} \mathrm{C}$ lab, for example, such parameters as the bounds of intervals and the maximum age. From the above reasoning, the FM is a fundamental parameter as the precision of measurements is directly dependent upon it. It is neces- 
sary to allow for an essential precondition of obtaining this FM. This parameter is suitable for an ideal case only, that is, for measurements without fluctuations of count efficiency and without additional fluctuations of the background.

\section{ACKNOWLEDGMENTS}

I am very obliged and thankful to my friends for their assistance, without which I could not have presented this paper. The figures in this paper were plotted with the AutoCAD program by my friend Vladimir Chapsky. I am very grateful to Leonid Margulyn for correcting the text.

\section{REFERENCES}

Crevecoeur, E. H., Stricht, A. V. and Capron, P. S. 1959 Precision of the dating method: Standardization of the calculation of the errors and the maximum age in the ${ }^{14} \mathrm{C}$ method. Science 45: 876-890.

Currie, L. A. 1972 The limit of precision in nuclear and analytical chemistry. Nuclear Instruments and Methods in Physics Research 100: 387-395.

Khait, V. Z. 1982 Errors of Radiometric Apparatus in a Definition of Radioactivity. Moscow, Academy of Sciences: 50-52 (in Russian).

Moljk, A., Drever, R. W. P. and Curran, F. R. S. 1957 The background of counters and radiocarbon dating. Proceedings of the Royal Society A 239: 433-445.

Ryabinin, A. L. 1978 Simple radiocarbon installation with the usage of integrated circuits. In Kocharov, G. E. and Dergachev. V. A., eds., Transactions of the 6th Scientific Society on the Problem of Astrophysical

Phenomena and Radiocarbon. Tbilisi, Tbilisi University: 267 (in Russian).

Sementsov, A. A. 1970 Stabilization of parameters of a scintillation installation for counting of natural radiocarbon. In Kocharov, G. E. and Dergachev. V. A., eds., Transactions of the Scientific Society on the Problem of Astrophysical Phenomena and Radiocarbon. Tbilisi, Tbilisi University: 75 (in Russian).

Vinogradov, A. P., Devirts, A. L., Dobkina, E. I. and Martishenko, N. G. 1961 A Definition of an Absolute ${ }^{14} \mathrm{C}$ Age with the Help of a Proportional Counter. Moscow, Academy of Sciences of the USSR (in Russian).

Zavelsky, F. S. 1972 About a precision and limits of the radiocarbon method of the definition of an absolute age. In Proceedings of the Academy of Sciences of the USSR, Geographical Series: 110-119 (in Russian). 